CLINICAL STUDY

\title{
Improvement of endothelial function with metformin and rosiglitazone treatment in women with polycystic ovary syndrome
}

\author{
Mojca Jensterle ${ }^{1}$, Miran Sebestjen ${ }^{2}$, Andrej Janez ${ }^{1}$, Janez Prezelj ${ }^{1}$, Tomaz Kocjan ${ }^{1}$, Irena Keber ${ }^{2}$ and Marija Pfeifer ${ }^{1}$ \\ Departments of ${ }^{1}$ Endocrinology, Diabetes and Metabolic Diseases and ${ }^{2}$ Angiology, University Medical Centre, Zaloška 7, SI-1000 Ljubljana, Slovenia \\ (Correspondence should be addressed to M Pfeifer; Email: misa.pfeifer@kclj.si)
}

\begin{abstract}
Objective: There is evidence of preclinical cardiovascular disease even in young women with polycystic ovary syndrome (PCOS). The aim of our study was to assess and compare the effects of metformin (MET) and rosiglitazone (ROSI) on endothelial function in PCOS patients.

Methods: For 6 months, 26 women with PCOS received either MET or ROSI. Blood samples for assessment of androgens, lipids, and high-sensitive C-reactive protein were taken at baseline and at endpoint. Endothelium-dependent flow-mediated dilation (FMD) and glyceryl trinitrate-induced endothelium-independent dilation of brachial artery were studied before and after treatment. Homeostasis model assessment $\left(\mathrm{HOMA}_{\mathrm{IR}}\right)$ calculation was applied as a measure of insulin resistance (IR).

Results: With treatment, FMD of brachial artery improved significantly from $4.2 \pm 6.6$ to $10.2 \pm 5.9 \%$ in MET group $(P=0.036)$ and from $2.9 \pm 3.2$ to $7.6 \pm 4.9 \%$ in ROSI group $(P=0.026)$, MET being as effective as ROSI $(P=0.70)$. The endothelium-independent dilation did not change. Additionally, administration of MET was associated with a significant decrease in $\operatorname{HOMA}_{\mathrm{IR}}(P=0.003)$, serum total and serum-free testosterone $(P=0.045$ and $P=0.008$ respectively $)$ and significantly higher frequencies of menstrual bleeding $(P=0.006)$.

Conclusions: A 6-month therapy with insulin sensitizers, MET and ROSI, resulted in marked improvement of endothelial function in young PCOS patients without clinically evident atherosclerosis who were not severely insulin resistant. Neither drug was superior to the other. We conclude that therapeutic intervention with either insulin sensitizer may reverse the atherosclerotic process in PCOS patients at its early stage.
\end{abstract}

European Journal of Endocrinology 159 399-406

\section{Introduction}

Polycystic ovary syndrome (PCOS) is a common endocrinopathy of complex and multifactorial etiology characterized by hyperandrogenism and chronic anovulation that affects $4-10 \%$ of women in reproductive age $(1,2)$. Over the last 20 years, it has been widely recognized that in addition to endocrine and reproductive abnormalities most women with PCOS demonstrate metabolic disturbances including insulin resistance (IR), type II diabetes mellitus, dyslipidemia, abdominal obesity, and metabolic syndrome (3-5).

In agreement with these metabolic derangements, there is increasing evidence of preclinical cardiovascular disease (CVD). Left ventricular hypertrophy (6), increased carotid intima-media thickness $(7-10)$, coronary artery calcification (11), and endothelial dysfunction $(7,12-14)$ have been found in obese and even in normal weight patients with PCOS. Clinically apparent CVD is much less evident in reproductive period of PCOS patients, although these women may have an increased incidence of CVD as they age (15).

Endothelial dysfunction is one of the preclinical manifestations of cardiovascular damage (16). It is the result of decreased endothelial production or increased degradation of nitric oxide (NO) (17) and is found in patients with coronary artery disease (18), in those with atherosclerotic risk factors (19), and in women with PCOS $(7,12-14)$. The healthy endothelium, particularly endothelium-derived NO, modulates the tone of the underlying vascular smooth muscle and inhibits several proatherogenic processes (20). Endothelial cell dysfunction is the initiating event in the development of atherosclerosis (21); therefore, the assessment of endothelial function has emerged as a tool for detection of preclinical CVD. Endothelial function can be measured as flow-mediated endothelium-dependent dilation (FMD) of the brachial artery using ultrasound.

One of the physiological actions of insulin in humans is to dilate skeletal muscle vasculature (22). The dilating 
effect of insulin is mediated by the endothelium-derived NO (23). IR may play a key role in the development of endothelial damage as it is associated with blunted endothelium-dependent, but not endothelium-independent dilation (24), with failure of hyperinsulinaemia to augment endothelium-dependent dilation (25). It is well known that metformin (MET) $(26,27)$ and rosiglitazone (ROSI) (28-33) improve IR, hyperinsulinism, and metabolic profile in PCOS, but their actual protection from cardiovascular morbidity and mortality has yet to be demonstrated.

The aim of our study was to assess and compare the effects of 6 months MET and ROSI administration on endothelial function in young women with PCOS. The novelty of this study is to explore whether there is a difference in the effects of the two insulin sensitizers on the endothelium in PCOS. The more potent drug would then be recommended to patients at higher cardiometabolic risk.

\section{Subjects and methods}

\section{Study population}

We recruited 28 women with PCOS as classified according to the National Institute of Child Health and Human Development (NICHD) criteria (34). We chose the NICHD criteria because they better define the population of PCOS patients that are at higher risk for IR and cardiometabolic complications than do the Rotterdam criteria (35). All subjects gave their written informed consent before entering the study that was conducted in accordance with the Declaration of Helsinki and approved by the national ethical committee. Clinical hyperandrogenism was defined by the presence of hirsutism, represented by a modified Ferriman-Gallwey score of 7 or more, persistence of acne during the third decade of life or later, or the presence of androgenic alopecia. No attempts were made to grade the severity of acne or alopecia. Hyperandrogenemia was defined as a total or free testosterone, androstenedione, and/or DHEA-sulfate (DHEAS) level above the 95th percentile of normal population values. Menstrual dysfunction was defined by more than six cycles with a length of more than 35 days (oligomenorrhoea), and/or when the patient had not had any menstrual bleeding for three consecutive months (amenorrhea) during the previous year. We took a history of menstrual bleeding for the period of 6 months before starting the treatment and followed each bleeding during the treatment. All patients had normal serum prolactin concentrations and thyroid function tests. Possible Cushing's syndrome or congenital (non-classic) adrenal hyperplasia were excluded when needed (34). Additional exclusion criteria were type I or type II diabetes mellitus, a significant cardiovascular or hepatic disease, and the use of medications known or suspected to affect reproductive or metabolic functions, within 60 days prior to study entry. None of the patients had ever taken insulin-sensitizing drugs prior to the study.

\section{Experimental protocol}

Patients were randomly allocated to a 6-month treatment with either MET $850 \mathrm{mg}$ twice daily or ROSI $4 \mathrm{mg}$ once a day. As a method of randomization, the RAND function in Excel was used. After the initial inclusion of 28 patients, 26 (93\%) finished the trial according to the protocol. Two women from ROSI group were excluded due to protocol violation. The MET group included 15 women (age 23.1 \pm 3.7 years). The ROSI group included 11 women (age $25.0 \pm 4.9$ years). They underwent hemodynamic testing at baseline and endpoint of the study. The endothelium-dependent FMD and the endothelium-independent (glyceryl trinitrate (GTN)-mediated) dilation of the brachial artery were measured in all subjects. A fasting blood was drawn for determination of glucose, insulin, and other observation parameters followed by a standard $75 \mathrm{~g}$ oral glucose tolerance test (OGTT). All the blood samples were centrifuged and the separated serum was kept frozen at $-40{ }^{\circ} \mathrm{C}$ until analyzed. In addition, safety parameters (hematology, liver and renal function, and serum electrolytes) were assessed before and at 2-month intervals during the study. Women were advised to use barrier contraception, instructed not to modify their usual eating habits and physical activity throughout the study, and asked to report any side-effects during the treatment. No heavy smokers were included in the study. All current smokers were requested to reduce the number of cigarettes smoked the week before and not to smoke at all $12 \mathrm{~h}$ before the hemodynamic study, to eliminate the acute effect of smoking on endothelial function. Smoking status of the patients during the study did not change, and there was a comparable proportion of smokers in either groups (four women in MET and three women in ROSI group).

\section{Assays}

Glucose levels were determined using a standard laboratory reference method (glucose oxidase method; Roche Hitachi 917). Luteinizing hormone (LH) and follicle-stimulating hormone (FSH) were determined using an immunometric assay (Diagnostic Products Corporation, Los Angeles, CA, USA). Androstenedione and DHEAS were measured by specific double antibody RIA using 125 I-labeled hormones (Diagnostic Systems Laboratories, Webster, TX, USA). Total and free testosterone levels were measured by coated tube RIA (DiaSorin, S. p. A, Salluggia, Italy, and Diagnostic Products Corporation respectively). Insulin was determined by IRMA (Biosource Europe S.A., Nivelles, Belgium). High-sensitive C-reactive protein (hsCRP) 
was measured by chemiluminescent immunoassay (Immulite, Diagnostic Products Corporation). Intraassay variations ranged from 1.6 to $6.3 \%$, and interassay variations ranged from 5.8 to $9.6 \%$ for the applied methods. Pre- and post-treatment samples from each patient were assayed in the same assay run.

\section{Assessment of IR}

Homeostasis model assessment (HOMA IR $)$ score calculation was applied as a measure for IR. The estimate of IR by HOMA $\mathrm{IR}_{\mathrm{IR}}$ score was calculated with the following formula: fasting serum insulin $(\mathrm{mU} / \mathrm{l}) \times$ fasting plasma glucose $(\mathrm{mmol} / \mathrm{l}) / 22.5$ (36). HOMA $\mathrm{IR}$ score values 2.0 were considered as a cutoff point for IR as published previously (37).

\section{Hemodynamic studies}

Endothelium-dependent FMD and GTN-induced (endothelium-independent) dilation of the brachial artery were studied using a high resolution B mode Advanced Technology Laboratories 5000 ultrasound system with a $7 \mathrm{MHz}$ linear array transducer, as described previously (18). The subjects rested in the supine position for $10 \mathrm{~min}$ before hemodynamic measurements were performed. The brachial artery was scanned in the longitudinal section $2-15 \mathrm{~cm}$ above the elbow to find the clearest images of the anterior and posterior wall layers. The mean arterial diameter was measured at the end of diastole, which was determined by simultaneous monitoring of the electrocardiogram. At least three cardiac cycles were analyzed for each scan and the measurements averaged. The flow velocity was measured at a fixed incident angle of $60^{\circ}$ to the vessel with the range gate of $1.5 \mathrm{~mm}$ located in the centre of the artery. The baseline (resting) blood flow was estimated by multiplying the velocity time integral of the Doppler flow signal (corrected for incident angle) by the vessel cross-sectional area. Hyperemic flow increase was induced by inflation of a blood pressure tourniquet, placed around the forearm, to a pressure of $300 \mathrm{mmHg}$ for $4.5 \mathrm{~min}$ inducing ischemia. Hyperemic flow was recorded for the first $15 \mathrm{~s}$ and diameter measurements were taken 45-60 s after cuff deflation. Increased blood flow caused shear stress induced increase in NO production and subsequent vasodilation. The endothelium-dependent dilation was expressed as the percentage change of the diameter after reactive hyperemia relative to the baseline diameter. A period of 10 min was allowed for vessel recovery, after which a further resting scan was taken. Endothelium-independent dilation was provoked by sublingual administration of $400 \mu \mathrm{g}$ of GTN, which acts as NO donor. The final scan was performed 4.5 min later. Endotheliumindependent dilation was expressed as the percentage change in the diameter after GTN administration relative to the baseline scan. The ratio between the endothelium-dependent and endothelium-independent dilation was calculated. All measurements were carried out by the same investigator who was blinded for the treatment assignment of the patients and also for the post- or pretreatment status, since there were more studies on endothelial dysfunction in PCOS going on simultaneously making that possible. To assess the reproducibility of measurements, 20 subjects were selected at random for repeated vascular studies. The correlation coefficient between the absolute differences and mean values of paired measurements was 0.92 , $P<0.05$, and the interclass correlation coefficient was $0.970, P<0.001$.

\section{Statistical analysis}

The variables showing a normal distribution, as determined by the Kolmogorov-Smirnov test, were expressed as means and s.D. HOMA $\mathrm{IR}_{\mathrm{I}}$ and hsCRP were highly skewed and were analyzed after logarithmic transformation. These data were expressed as median and quartile range. Differences between groups before and after the intervention were tested for significance by Student's $t$-test for unpaired data for normally distributed variables. The change of each variable after the intervention period was tested by Student's $t$-test for paired variables in each group. For correlation analysis, Pearson's correlation coefficient was calculated for normally distributed variables. The criterion for statistical significance was a $P$ value of less than 0.05 . All calculations were performed with the Statistica (data analysis software system), version 7.1 (StatSoft Inc., 2005, Tulsa, OK, USA).

\section{Results}

\section{Baseline characteristics}

The mean \pm s.D. values for the normally distributed parameters and median with quartile range of HOMA $\mathrm{AR}_{\mathrm{IR}}$ and hsCRP, at baseline are reported in Table 1. As expected because of randomization, there were no significant differences in any of the parameters between the treatment groups at baseline $(P$ values not reported). Patients showed the characteristic dyslipidemia of PCOS with low high density lipoprotein cholesterol levels.

\section{Changes in anthropometric and circulating variables}

Only minor and statistically non-significant changes were observed in the body mass index (BMI) and waist circumference values in both groups after treatment intervention (Table 1). OGTT did not reveal an impaired glucose tolerance (IGT) in any of the patients studied in the ROSI group (neither at baseline nor at endpoint). 
Table 1 Polycystic ovary syndrome (PCOS) patients' characteristics (mean and s.D. (median and quartile range)) at baseline and after 6 months of metformin or rosiglitazone treatment.

\begin{tabular}{|c|c|c|c|c|c|c|c|c|}
\hline \multirow[b]{3}{*}{ Characteristics } & \multicolumn{4}{|c|}{ Metformin group $(n=15)$} & \multicolumn{4}{|c|}{ Rosiglitazone group $(n=11)$} \\
\hline & \multicolumn{2}{|c|}{ Baseline } & \multicolumn{2}{|c|}{ Endpoint } & \multicolumn{2}{|c|}{ Baseline } & \multicolumn{2}{|c|}{ Endpoint } \\
\hline & $\begin{array}{c}\text { Mean } \\
\text { (median) }\end{array}$ & $\begin{array}{c}\text { S.D. } \\
\text { (quartile range) }\end{array}$ & $\begin{array}{c}\text { Mean } \\
\text { (median) }\end{array}$ & $\begin{array}{c}\text { S.D. } \\
\text { (quartile range) }\end{array}$ & $\begin{array}{c}\text { Mean } \\
\text { (median) }\end{array}$ & $\begin{array}{c}\text { S.D. } \\
\text { (quartile range) }\end{array}$ & $\begin{array}{c}\text { Mean } \\
\text { (median) }\end{array}$ & $\begin{array}{c}\text { S.D. } \\
\text { (quartile range) }\end{array}$ \\
\hline BMI $\left(\mathrm{kg} / \mathrm{m}^{2}\right)$ & 29.6 & 6.9 & 29.0 & 6.8 & 28.8 & 8.8 & 28.8 & 8.1 \\
\hline Waist circumference $(\mathrm{cm})$ & 90.8 & 14.7 & 90.1 & 14.4 & 88.7 & 18.7 & 89.4 & 19.5 \\
\hline BP systolic (mmHg) & 121.3 & 13.6 & 112.3 & 14.6 & 122.3 & 19.4 & 111.4 & 15.2 \\
\hline BP dyastolic (mmHg) & 75.0 & 9.6 & 75.7 & 12.9 & 76.8 & 13.8 & 75.0 & 11.2 \\
\hline Fasting glucose $(\mathrm{mmol} / \mathrm{l})$ & 4.51 & 0.56 & 4.32 & 0.39 & 4.37 & 0.30 & 4.37 & 0.20 \\
\hline Fasting insulin (mIU/l) & 14.33 & 7.66 & 7.03 & 4.06 & 13.15 & 6.31 & 6.73 & 4.60 \\
\hline $\mathrm{HOMA}_{\mathrm{IR}}$ score & 2.98 & 3.17 & 1.24 & $1.54^{\ddagger}$ & 2.72 & 1.58 & 1.64 & 1.93 \\
\hline $\mathrm{LH}(\mathrm{IU} / \mathrm{l})$ & 13.30 & 15.87 & 9.94 & 7.62 & 10.79 & 7.23 & 8.28 & 5.93 \\
\hline FSH (IÚ/l) & 5.84 & 2.08 & 6.37 & 2.95 & 5.81 & 1.39 & 4.84 & 2.64 \\
\hline DHEA'S $(\mu \mathrm{mol} / \mathrm{l})$ & 5.66 & 1.63 & 5.70 & 1.55 & 7.01 & 2.53 & 7.34 & 3.18 \\
\hline Androstenedione $(\mathrm{nmol} / \mathrm{l})$ & 7.75 & 2.74 & 8.33 & 3.49 & 9.72 & 2.06 & 11.04 & 2.63 \\
\hline Serum total testosterone $(\mathrm{nmol} / \mathrm{l})$ & 2.41 & 0.80 & 1.98 & $0.65^{\star}$ & 3.02 & 1.22 & 2.95 & 1.06 \\
\hline Serum-free testosterone $(\mathrm{pmol} / \mathrm{l})$ & 9.75 & 4.32 & 7.03 & $4.07^{\dagger}$ & 11.04 & 6.55 & 10.13 & 3.73 \\
\hline Cholesterol $(\mathrm{mmol} / \mathrm{l})$ & 4.61 & 0.89 & 4.59 & 0.93 & 5.05 & 0.81 & 5.41 & 1.12 \\
\hline HDL Cholesterol (mmol/l) & 1.27 & 0.27 & 1.29 & 0.25 & 1.35 & 0.33 & 1.38 & 0.30 \\
\hline LDL Cholesterol (mmol/l) & 2.89 & 0.80 & 2.87 & 0.84 & 3.17 & 0.81 & 3.35 & 0.99 \\
\hline Triglyceride $(\mathrm{mmol} / \mathrm{l})$ & 0.99 & 0.50 & 0.98 & 0.62 & 1.17 & 0.43 & 1.48 & 0.88 \\
\hline $\mathrm{hsCRP}(\mathrm{mg} / \mathrm{dl})$ & 1.80 & 7.22 & 1.92 & 6.18 & 1.09 & 3.21 & 0.64 & 1.67 \\
\hline Periods/subject per 6 month & 2.93 & 1.98 & 4.07 & $2.05^{\dagger}$ & 3.45 & 1.85 & 4.45 & 1.37 \\
\hline
\end{tabular}

The treatment/time effect versus baseline within the groups, tested by the Student's $t$-test for paired variables: ${ }^{*} P<0.05,{ }^{\dagger} P<0.01,{ }^{\ddagger} P<0.005$. All significant changes are in bold. The Student's $t$-test for unpaired data for the differences between the groups, $P=\mathrm{NS}$. BMI, body mass index: BP, blood pressure; $\mathrm{HOMA}_{\mathrm{IP}}$ score, homeostasis model assessment of insulin resistance; $\mathrm{LH}$, luteinizing hormone; $\mathrm{FSH}$, folliclestimulating hormone; DHEAS DHEA sulfate; hsCRP, high-sensitive C-reactive protein. The medians and quartile ranges are written in italics. 
In the MET group, two patients were found to have IGT at baseline. After treatment, the IGT was still observed in the two patients. HOMA $\mathrm{IR}_{\mathrm{IR}}$ score values significantly decreased in MET group $(P=0.003$ for the treatment/ time effect). There was a non-significant tendency of HOMA $_{\mathrm{IR}}$ improvement in ROSI group $(P=0.15)$ that was predominantly driven by a decrease in fasting insulin levels. The delta change in $\mathrm{HOMA}_{\mathrm{IR}}$ between MET and ROSI group was not statistically significant $(P=0.283)$. Administration of MET was associated with a significant decrease in serum total and free testosterone $(P=0.045$ and $P=0.008$ respectively). Serum total and free testosterone decreased after treatment with ROSI, but this was not statistically significant $(P=0.85$ and 0.58 respectively). No statistically significant change over time occurred in the remaining laboratory variables (fasting glucose, fasting insulin, total, high density lipoprotein (HDL) and low density lipoprotein (LDL) cholesterol, triglycerides, LH, FSH, LH/FSH ratio, androstenedione, DHEAS, and hsCRP; Table 1).

\section{Changes in menstrual pattern}

Both treatment interventions resulted in an improvement of patients' menstrual pattern. Menstrual frequency increased from 0.57 to 0.76 cycles per month on average in the MET group $(P=0.006)$ and from 0.67 to 0.81 per month $(P=0.076)$ in the ROSI group (Table 1).

\section{Hemodynamic studies}

No differences were observed in the baseline diameters of the brachial artery, FMD, and GTN-induced dilation between groups. After treatment, FMD of the brachial artery improved significantly in each group (Table 2). The treatment effect was comparable in both groups. No difference was observed in GTN-induced dilation before and after treatment in either group (Table 2), with no difference between the groups. The ratio of FMD to GTN-mediated dilation significantly increased in both groups (Table 2), with no difference between the groups. There was a negative correlation between the change in FMD (\%) and the change in testosterone with MET treatment $(P=0.05, R=-0.52)$.

Both drugs were well tolerated and a few minor adverse events did not lead to discontinuation of the treatment in any of the patients. In the MET group, two subjects had temporary mild gastrointestinal problems. No elevation of liver enzymes was found and no case of edema was reported in the ROSI group.

\section{Discussion}

We demonstrated that both MET and ROSI administration improved endothelial function in young women with PCOS, MET being as effective as ROSI. The augmentation of the vascular reactivity was accompanied by a clinically meaningful improvement in menstrual pattern in both treatment groups and by a significant reduction in $\mathrm{HOMA}_{\mathrm{IR}}$ and serum total and serum-free testosterone concentration in the MET group. To our knowledge, this is the first study to compare the effects of both insulin sensitizers on arterial reactivity in young patients with PCOS.

In the present trial, FMD of the brachial artery improved significantly after MET and ROSI administration, while there was no effect on GTN-induced dilation in either group. The ratio of FMD to GTN-induced dilation, as defined by Zeiher et al. (38), increased significantly in both treatment groups indicating that the brachial artery dilation improved exclusively due to the improvement of the endothelial function and not because of the better vascular smooth muscle cell activity.

To date, interventions with MET have brought inconclusive results regarding the endothelial function in PCOS. Its administration has been proven beneficial in few recent studies: endothelial dysfunction was reversed to control levels in 20 PCOS patients (39)

Table 2 Brachial artery endothelium-dependent FMD and endothelium-independent GTN dilation before and after 6 months of metformin or rosiglitazone treatment.

\begin{tabular}{|c|c|c|c|c|c|}
\hline \multirow[b]{2}{*}{ Characteristics } & \multicolumn{2}{|c|}{ Baseline } & \multicolumn{2}{|c|}{ Endpoint } & \multirow[b]{2}{*}{$P$ value } \\
\hline & Mean & S.D. & Mean & S.D. & \\
\hline \multicolumn{6}{|l|}{ Metformin group $(n=15)$} \\
\hline Baseline artery diameter $(\mathrm{mm})$ & 4.67 & 3.21 & 5.68 & 4.28 & NS \\
\hline FMD (\% of change) & 4.2 & 6.6 & 10.2 & 5.9 & 0.036 \\
\hline GTN (\% of change) & 21.9 & 8.2 & 21.3 & 5.4 & NS \\
\hline FMD/GTN & 0.12 & 0.29 & 0.46 & 0.23 & 0.003 \\
\hline \multicolumn{6}{|l|}{ Rosiglitazone group $(n=11)$} \\
\hline Baseline artery diameter (mm) & 3.99 & 2.09 & 3.09 & 1.57 & NS \\
\hline FMD (\% of change) & 2.9 & 3.2 & 7.6 & 4.9 & 0.026 \\
\hline GTN (\% of change) & 18.4 & 3.8 & 17.9 & 6.1 & NS 0 \\
\hline FMD/GTN & 0.15 & 0.18 & 0.41 & 0.24 & 0.019 \\
\hline
\end{tabular}

FMD, flow-mediated dilation; GTN, glyceryl trinitrate-induced dilation; NS, non significant. Bold indicates $P$ values with significant change of the studied parameters with treatment. 
and improved in 30 non-dyslipidemic, non-hypertensive, young, normal weight women with PCOS, 6 months after MET treatment (40). On the other hand, endothelial dysfunction did not improve after 3 months of MET treatment in another group of PCOS patients (41). Similarly, in a larger controlled trial, the improvement of IR with 6 months of MET did not induce an increase in FMD (42). There are even more limited data on the efficacy of ROSI on the endothelial function: ROSI was shown to improve endothelial dysfunction in non-obese young women with PCOS in only one study (43). However, there are some studies suggesting an improvement of endothelial dysfunction in patients with newly diagnosed type II diabetes and coronary artery disease with another thiazolidinedione, pioglitazone (44). The improvement was independent from changes in insulin sensitivity.

The mechanism by which MET and ROSI improve endothelial function in PCOS is still unclear; however, an improvement in insulin sensitivity seems to be the most important factor (40). Phosphatidylinositol 3-kinasedependent insulin-signaling pathways in endothelium related to production of NO share striking similarities with metabolic pathways in skeletal muscle that promote glucose uptake. Other distinct non-metabolic branches of insulin-signaling (MAP-kinase) pathways regulate secretion of the vasoconstrictor ET-1 in endothelium. Metabolic IR is characterized by pathway-specific impairment in phosphatidylinositol 3-kinase-dependent signaling, which in endothelium may cause imbalance between the production of NO and secretion of ET-1 (45). Increased ET-1 levels have already been demonstrated in PCOS population (46). Furthermore, IR is linked to the endothelial dysfunction also by other mechanisms, such as increased oxidative stress, increased activity of the rennin-angiotensin system and the action of hormones and cytokines secreted by the adipose tissue $(47,48)$. Increased vascular stiffness and impaired vasodilatory action of insulin $e x$ vivo were demonstrated in patients with PCOS suggesting an abnormal insulin-regulated endothelial NO production in the vasculature (14). Endothelial dysfunction in young women with PCOS was associated with IR also in cross-sectional $(12,13)$ and interventional studies $(40,43)$. Significant decrease in IR after 6 months of MET treatment was accompanied by the improvement in endothelial structure and function in young, normal weight women with PCOS (40), suggesting an important role of IR and hyperinsulinaemia in the precocious atherogenesis in PCOS patients. Our data support these latter findings. A significant association between beneficial effect of ROSI on endothelial function and an improvement in insulin sensitivity was also reported in PCOS (43).

Alternatively, the obvious beneficial effect of MET and ROSI on endothelial function could be mediated by additional mechanisms. In our study, total and free testosterone concentrations decreased significantly after MET and tended to be reduced after ROSI therapy.
The decrease in testosterone levels correlated with the improvement of FMD in the MET group. Similarly, in another study, androgenic profile improved along with the improvement in endothelial structure and function after 6-month MET administration in PCOS women (40). A positive correlation between abnormal endothelial function and testosterone levels was found in hyperandrogenic insulin-resistant women with PCOS, an association that was stronger than that with IR (12). Since androgen receptors are present on the arterial wall, the direct effect of androgens on the vasculature cannot be excluded and the reduction of hyperandrogenemia might improve vascular reactivity (49). However, the role of different androgens in cardiovascular physiology and disease remains unclear and whether hyperandrogenism has protective or deleterious effects on atherogenesis in women with PCOS remains to be clarified.

Accumulating evidence suggests that inflammatory markers like hsCRP directly promote atherosclerotic processes and endothelial cell inflammation leading to atherothrombosis $(50,51)$. In our study, a decrease in hsCRP cannot be included in the parameters affecting the improvement of endothelial function, since significant decreases in hsCRP were not detected after treatment in either group. On the other hand, Tarkun et al. (43) proved beneficial effects of ROSI on endothelial function and hsCRP in normal weight young women with PCOS.

Furthermore, reduction of body weight cannot explain the improvement of endothelial function in our study, since BMI did not change with treatment. It has been shown previously that endothelial function in PCOS does not exclusively depend on BMI. Impairment of endothelial structure and function was found even in normal weight women with PCOS (52). Therefore, MET and ROSI can be used to improve the cardiovascular risk also in lean women with PCOS.

Additionally, the improvement of endothelial function in our patients was not associated with any change in the characteristic dyslipidemic pattern of PCOS (low HDL cholesterol) in either therapeutic group. In this regard, FMD improved with ROSI treatment in PCOS without an associated change in the lipid profile (43). On the contrary, the endothelial function improved with MET intervention in PCOS in association with an increase in HDL and a reduction in LDL cholesterol (40). However, the implied mechanisms of insulinsensitizing treatment effects on endothelial function in PCOS are still a matter of debate.

At this point, the limitations of our study should be mentioned: this was an intervention study with relatively small sample size where the endothelial function was not additionally assessed by biochemical methods (ET-1 and cell adhesion molecules were not measured). Additionally, we did not measure FMD in a healthy BMI-matched control group to compare the post-treatment FMDs of our patients to, which would have enabled us to see whether the FMD values had normalized. Nevertheless, from the clinical point of 
view, the significant improvement of endothelial function in our patients with both insulin sensitizers is the most relevant finding. If FMD has normalized in 6 months or not (yet) seems to be of less importance.

Less significant effects of ROSI when compared with MET on some other studied parameters might reflect a type 2 error as, also due to dropouts, fewer subjects were studied in ROSI group.

The advantage of our study was that we chose young PCOS patients without any clinical signs of atherosclerosis who were not severely insulin resistant (as assessed by $\mathrm{HOMA}_{\mathrm{IR}}$ ), which enabled us to demonstrate that therapeutic interventions with insulin sensitizers may reverse the atherosclerotic process in young women in its early stage. It seems prudent to plan long-term therapy that addresses the reduction of $\mathrm{CV}$ risk in PCOS (53). However, regarding translation of these data to clinical use in PCOS, it needs to be acknowledged that, because of an uncertain safety profile in pregnancy, ROSI should not be prescribed to women wishing to conceive. Alternatively, a number of studies have confirmed apparent safety of MET, without teratogenicity and even with a potential for reducing the risk of miscarriage and gestational diabetes in women with PCOS (54).

In conclusion, the 6-month treatment with ROSI and MET of young PCOS women resulted in marked improvement in endothelial function, MET being as effective as ROSI. Further randomized controlled trials with larger numbers of patients are required to determine whether insulin-sensitizing therapy can be useful in reducing cardiovascular risk in women with PCOS long term.

\section{Declaration of interest}

The authors declare that there is no conflict of interest that would prejudice the impartiality of this scientific work.

\section{Funding}

Fellowships/Grants: This study was supported by Slovenian Research Agency, Research Program No. P3-0298.

\section{Acknowledgements}

We are grateful to Alenka Roš, RN, for assistance with the assay procedures and for performing anthropometric measurements.

\section{References}

1 Franks S. Polycystic ovary syndrome. New England Journal of Medicine 1995333 853-856.

2 Azziz R, Woods KS, Reyna R, Key TJ, Knochenhauer ES \& Yildiz BO. The prevalence and features of the polycystic ovary syndrome in an unselected population. Journal of Clinical Endocrinology and Metabolism 200489 2745-2749.

3 Dunaif A, Segal KR, Futterweit W \& Dobrjansky A. Profound peripheral insulin resistance, independent of obesity, in polycystic ovary syndrome. Diabetes 1989381165.
4 Legro RS, Kunselman AR \& Dunaif A. Prevalence and predictors of dyslipidemia in women with polycystic ovary syndrome. American Journal of Medicine 2001111 607-613.

5 Lo JC, Feigenbaum SL, Yang J, Pressman AR, Selby JV \& Go AS. Epidemiology and adverse cardiovascular risk profile of diagnosed polycystic ovary syndrome. Journal of Clinical Endocrinology and Metabolism 200691 1357-1363.

6 Orio F Jr, Palomba S, Spinelli L, Cascella T, Tauchmanovà L, Zullo F, Lombardi G \& Colao A. The cardiovascular risk of young women with polycystic ovary syndrome: an observational, analytical, prospective case-control study. Journal of Clinical Endocrinology and Metabolism 200489 3696-3701.

7 Orio F Jr, Palomba S, Cascella T, De Simone B, Di Biase S, Russo T, Labella D, Zullo F, Lombardi G \& Colao A. Early impairment of endothelial structure and function in young normal-weight women with polycystic ovary syndrome. Journal of Clinical Endocrinology and Metabolism 200489 4588-4593.

8 Vryonidou A, Papatheodorou A, Tavridou A, Terzi T, Loi V, Vatalas IA, Batakis N, Phenekos C \& Dionyssiou-Asteriou A. Association of hyperandrogenemic and metabolic phenotype with carotid intima-media thickness in young women with polycystic ovary syndrome. Journal of Clinical Endocrinology and Metabolism $2005902740-2746$.

9 Lakhani K, Hardiman P \& Seifalian AM. Intima-media thickness of elastic and muscular arteries of young women with polycystic ovaries. Atherosclerosis 2004175 353-359.

10 Talbott EO, Guzick DS, Sutton-Tyrrell K, McHugh-Pemu KP, Zborowski JV, Remsberg KE \& Kuller LH. Evidence for association between polycystic ovary syndrome and premature carotid atherosclerosis in middle-aged women. Arteriosclerosis, Thrombosis, and Vascular Biology $2000202414-2421$.

11 Shroff R, Kerchner A, Maifeld M, Van Beek EJ, Jagasia D \& Dokras A. Young obese women with polycystic ovary syndrome have evidence of early coronary atherosclerosis. Journal of Clinical Endocrinology and Metabolism 200792 4609-4614.

12 Paradisi G, Steinberg HO, Hempfling A, Cronin J, Hook G, Shepard MK \& Baron AD. Polycystic ovary syndrome is associated with endothelial dysfunction. Circulation 2001103 1410-1415.

13 Tarkun I, Arslan BC, Cantürk Z, Türemen E, Sahin T \& Duman C. Endothelial dysfunction in young women with polycystic ovary syndrome: relationship with insulin resistance and low-grade chronic inflammation. Journal of Clinical Endocrinology and Metabolism $2004895592-5596$.

14 Kelly CJG, Speirs A, Gould GW, Petrie JR, Lyall H \& Connell JMC. Altered vascular function in young women with polycystic ovary syndrome. Journal of Clinical Endocrinology and Metabolism 2002 $87742-746$.

15 Azziz A. Long term morbidity of PCOS. In The Polycystic Ovary Syndrome: Current Concepts on Pathogenesis and Clinical Care, $\mathrm{p} 124$. Ed. R Azziz, New York: Springer Science+Business Media, LCC, 2007.

16 Bonetti PO, Lerman LO \& Lerman A. Endothelial dysfunction: a marker of atherosclerotic risk. Arteriosclerosis, Thrombosis, and Vascular Biology 200323 168-175.

17 Celermajer DS. Endothelial dysfunction: does it matter? Is it reversible? Journal of the American College of Cardiology 199730 325-333.

18 Celermajer DS, Sorensen KE, Gooch VM, Spiegelhalter DJ, Miller OI, Sullivan ID, Lloyd JK \& Deanfield JE. Non-invasive detection of endothelial dysfunction in children and adults at risk of atherosclerosis. Lancet 1992340 1111-1115.

19 Chauhan A, Mullins PA, Taylor G, Petch MC \& Schofield PM. Both endothelium-dependent and endothelium-independent function is impaired in patients with angina pectoris and normal coronary angiograms. European Heart Journal 199718 60-68.

20 Abdu TAM, Elhadd T, Pfeifer M \& Clayton RN. Endothelial dysfunction in endocrine disease. Trends in Endocrinology and Metabolism 200112 257-265.

21 Ross R. Atherosclerosis: an inflammatory disease. New England Journal of Medicine $19993 \mathbf{3 4 0} 115-126$. 
22 Laakso M, Edelman SV, Brechtel G \& Baron AD. Decreased effect of insulin to stimulate skeletal muscle blood flow in obese man. A novel mechanism for insulin resistance. Journal of Clinical Investigation $1990 \mathbf{8 5} 1844-1852$.

23 Scherrer U, Randin D, Vollenweider P, Vollenweider L \& Nicod P. Nitric oxide release accounts for insulin's vascular effects in humans. Journal of Clinical Investigation 199494 2511-2515.

24 Arcaro G, Zamboni M, Rossi L, Turcato E, Covi G, Armellini F, Bosello $\mathrm{O} \&$ Lechi A. Body fat distribution predicts the degree of endothelial dysfunction in uncomplicated obesity. International Journal of Obesity and Related Metabolic Disorders 199923 936-942.

25 Steinberg HO, Chaker H, Leaming R, Johnson A, Brechtel G \& Baron AD. Obesity/insulin resistance is associated with endothelial dysfunction. Implications for the syndrome of insulin resistance. Journal of Clinical Investigation $1996972601-2610$.

26 Harborne L, Fleming R, Lyall H, Norman J \& Sattar N. Descriptive review of the evidence for the use of metformin in polycystic ovary syndrome. Lancet 2003361 1894-1901.

27 Lord JM, Flinght IH \& Norman RJ. Metformin in polycystic ovary syndrome: systematic review and meta-analysis. BMJ 2003327 951-953.

28 Cataldo NA, Abbasi F, McLaughlin TL, Lamendola C \& Reaven GM. Improvement in insulin sensitivity followed by ovulation and pregnancy in a woman with polycystic ovary syndrome who was treated with rosiglitazone. Fertility and Sterility $2001 \mathbf{7 6}$ 1057-1059.

29 Ghazeeri G, Kutteh WH, Bryer-Ash M, Haas D \& Ke RW. Effect of rosiglitazone on spontaneous and clomiphene citrate-induced ovulation in women with polycystic ovary syndrome. Fertility and Sterility $2003 \mathbf{7 9} 562-566$.

30 Sepilian V \& Nagamani M. Effects of rosiglitazone in obese women with polycystic ovary syndrome and severe insulin resistance. Journal of Clinical Endocrinology and Metabolism 200590 60-65.

31 Cataldo NA, Abbasi F, McLaughlin TL, Basina M, Fechner PY, Giudice LC \& Reaven GM. Metabolic and ovarian effects of rosiglitazone treatment for 12 weeks in insulin-resistant women with polycystic ovary syndrome. Human Reproduction 200621 109-120.

32 Rautio K, Tapanainen JS, Ruokonen A \& Morin-Papunen LC. Endocrine and metabolic effects of rosiglitazone in overweight women with PCOS: a randomized placebo-controlled study. Human Reproduction 200621 1400-1407.

33 Majuri A, Santaniemi M, Rautio K, Kunnari A, Vartiainen J, Ruokonen A, Kesaniemi YA, Tapanainenm JS, Ukkola O \& MorinPapunen L. Rosiglitazone treatment increases plasma levels of adiponectin and decreases levels of resistin in overweight women with PCOS: a randomized placebo-controlled study. European Journal of Endocrinology $2007156263-269$.

34 Zawadzki JK \& Dunaif A. Diagnostic criteria for polycystic ovary syndrome. In Polycystic Ovary Syndrome, pp 377-384. Eds A Dunaif, F Haseltine \& GR Merriam, Boston: Blackwell Scientific Publications, 1992

35 Azziz R. Definition, diagnosis, and epidemiology of the polycystic ovary syndrome. In The Polycystic Ovary Syndrome: Current Concepts on Pathogenesis and Clinical Care, p 5. Ed. R Azziz, New York: Springer Science+Business Media, LCC, 2007.

36 Matthews DR, Hosker JP, Rudenski AS, Naylor BA, Treacher DF \& Turner RC. Homeostasis model assessment: insulin resistance and beta-cell function from fasting plasma glucose and insulin concentrations in man. Diabetologia $1985 \mathbf{2 8} 412-419$.

37 Hedblad B, Nilsson P, Janzon L \& Berglund G. Relation between insulin resistance and carotid intima-media thickness and stenosis in non-diabetic subjects. Results from a cross-sectional study in Malmo, Sweden. Diabetic Medicine 200017 299-307.

38 Zeiher AM, Schächinger V \& Minners J. Long-term cigarette smoking impairs endothelium-dependent coronary arterial vasodilator function. Circulation 199592 1094-1100.
39 Diamanti-Kandarakis E, Alexandraki K, Protogerou A, Piperi C, Papamichael C, Aessopos A, Lekakis J \& Mavrikakis M. Metformin administration improves endothelial function in women with polycystic ovary syndrome. European Journal of Endocrinology 2005 52 749-756.

40 Orio F Jr, Palomba S, Cascella T, De Simone B, Manguso F, Savastano S, Russo T, Tolino A, Zullo F, Lombardi G, Azziz R \& Colao A. Improvement in endothelial structure and function after metformin treatment in young normal-weight women with polycystic ovary syndrome: results of a 6-month study. Journal of Clinical Endocrinology and Metabolism $2005906072-6076$.

41 Lowenstein L, Damti A, Pillar G, Shott S \& Blumenfeld Z. Evaluation of endothelial function in women with polycystic ovary syndrome. European Journal of Obstetrics, Gynecology, and Reproductive Biology $2007134208-212$.

42 Meyer C, McGrath BP \& Teede HJ. Effects of medical therapy on insulin resistance and the cardiovascular system in polycystic ovary syndrome. Diabetes Care $200730471-478$.

43 Tarkun I, Cetinarslan B, Türemen E, Sahin T, Cantürk Z \& Komsuoglu B. Effect of rosiglitazone on insulin resistance, C-reactive protein and endothelial function in non-obese young women with polycystic ovary syndrome. European Journal of Endocrinology 2005153 115-121.

44 Sourij H, Zweiker R \& Wascher TC. Effects of pioglitazone on endothelial function, insulin sensitivity, and glucose control in subjects with coronary artery disease and new-onset type 2 diabetes. Diabetes Care 200629 1039-1045.

45 Kim J, Montagnani M, Koh KK \& Quon MJ. Reciprocal relationships between insulin resistance and endothelial dysfunction. Circulation $20061131888-1904$.

46 Diamanti-Kandarakis E, Spina G, Kouli C \& Migdalis I. Increased endothelin-1 levels in women with polycystic ovary syndrome and the beneficial effect of metformin therapy. Journal of Clinical Endocrinology and Metabolism 200186 4666-4673.

$47 \mathrm{Ki}$-Jarvinen H. Insulin resistance and endothelial dysfunction. Best Practice and Research. Clinical Endocrinology and Metabolism 2003 17 411-430.

48 Seligman B, Biolo A, Polanczyk C, Gross J \& Clausell N. Increased plasma levels of endothelin 1 and von Willebrand factor in patients with type 2 diabetes and dyslipidemia. Diabetes Care 200023 1395-1400.

49 Landin K, Tengborn L \& Smith U. Treating insulin resistance in hypertension with metformin reduces both blood pressure and metabolic risk factors. Journal of Internal Medicine 1991229 181-187.

50 Pasceri V, Willerson JT \& Yeh ET. Direct proinflammatory effect of C-reactive protein on human endothelial cells. Circulation 2000 102 2165-2168.

51 Sjöholm A \& Nyström T. Endothelial inflammation in insulin resistance. Lancet $2005365610-612$.

52 Orio F Jr, Palomba S, Cascella T, De Simone B, Di Biase S, Russo T, Labella D, Zullo F, Lombardi G \& Colao A. Early impairment of endothelial structure and function in young normal-weight women with polycystic ovary syndrome. Journal of Clinical Endocrinology and Metabolism 200489 4588-4593.

53 Nestler JE. Metformin for the treatment of the polycystic ovary syndrome. New England Journal of Medicine 2008358 47-54.

54 Balen A. Strategies for ovulation induction in the management of anovulatory polycystic ovary syndrome. In The Polycystic Ovary Syndrome: Current Concepts on Pathogenesis and Clinical Care, p 110. Ed. R Azziz, New York: Springer Science+Business Media, LCC, 2007.

Received 30 June 2008

Accepted 19 July 2008 